\title{
Wavelet Analysis on Solar Wind Parameters and Geomagnetic Indices
}

\author{
Ch. Katsavrias ${ }^{1}$, P. Preka-Papadema ${ }^{1}$, X. \\ Moussas $^{1}$
}

(C) Springer $\bullet \bullet \bullet$

\begin{abstract}
The sun as an oscillator produces frequencies which propagate in the heliosphere, via solar wind, to the terrestrial magnetosphere. We searched for those frequencies in the parameters of the near Earth solar plasma and the geomagnetic indices for the past four solar cycles. The solar wind parameters used in this work are the interplanetary magnetic field, plasma beta, Alfven Mach number, solar wind speed, plasma temperature, plasma pressure, plasma density and the geomagnetic indices $D_{S T}$, AE, $A_{p}$ and $K_{p}$. We found out that each parameter of the solar wind exhibit certain periodicities which differentiate in each cycle. Our results indicate intermittent periodicities in our data, some of them shared between the solar wind parameters and geomagnetic indices.
\end{abstract}

Keywords: Magnetosphere, Geomagnetic Disturbances, Solar Cycle, Observations, Solar Wind, Disturbances , Magnetic fields, Interplanetary, Wavelet

\section{Introduction}

Solar activity is quantified, mostly, by sunspot (or Wolf) number and exhibits a basic 11 years periodicity with 5.5 and 3.7 years harmonics (Currie, 1976; Carta et al., 1982; Diurovic and Paquet, 1996; Mursula. Usoskin. and Zieger, 1997; Polvgiannakis. Preka-Papadema, and Moussas, 2003)). The quasi-biennial oscillation and the 1.3 years periodicity, short-term periodicities of 154,128 , $102,77,51$ and 27 days have also been observed in the number of sunspots as well as in solar energetic events such as flares (Kilic, 2009) and coronal mass ejections (Lou et al., 2003). The identification, interpretation and modelling of solar periodicities is an open research issue:

- The 11 years periodicity in the mumber of sunspots and other solar activity (Djurovic and Paquet, 1996; Carta et al., 1982; Mursula, Usoskin, and Zieger,

\footnotetext{
${ }^{1}$ Department of Physics of Astrophysics, Astronomy and Mechanics, Department of Physics, University of Athens, Panepistimiopolis Zografos (Athens), GR-15783, Greece email: ckatsavrias@phys.uoa.gr email: ppreka@phys.uoa.gr email: xmoussas@phys.uoa.gr
} 
1997), is believed to originate from magnetohydrodynamic dynamo action at the base of the convection zone.

- Based on magnetic helicity observations, Bao and Zhang (1998), proposed a double-cycle solar magnetic dynamo model with one dynamo operating at the base of the convection zone (11-year cycle) due to radial shear and the other at the top of the convection zone (quasi-biennial periodicity) due to latitudal shear (Benevolenskava, 1998, 2000).

- It has also been proposed that the sunspot number can be well fitted by a superposition of the usual 11-yr cycles and wave trains with periodicity continuously varying from 3 years at solar maximum, to 1.7 years towards solar minimum (Polygiannakis. Preka-Papadema, and Moussas, 2003).

- An 154-day periodicity was observed in the solar flare rate, the $10.7 \mathrm{~cm}$ radio flux, sunspot number and the global magnetic field of the Sun (Clúa de Gonzalez et al. 1993; Lean and Brueckner, 1989; Cane. Richardson. and von Rosenvinge, 1998). Bai and Sturrock (1993), interpret this periodicity (51, 77, 102 and 128 days as well) as sub harmonics of a solar clock mechanism with a fundamental of 25.5 days. On the other hand, Rieger et al. (1984) proposed the g-mode oscillations as a possible cause while Wolff (1992) a combination of two r-modes with an interior g-mode (see also Dimitropoulou, Moussas, and Strintzi, 2008, 2009, for a discussion on Rossby wave origin of the Rieger periodicities).

- The 1.3 years oscillation was found in solar magnetic field during the maxima and declining phase of 8 solar cycles. This is thought to be generated at the tachocline as an inherent feature of the global magnetic field (Obridko and Shelting, 2007; Howe et al., 2000). The same periodicity appears in sunspot number and other solar parameters; Krivova and Solanki (2002) interprete it, as a sub harmonic, of the 154 days period mentioned above.

- A wavelet analysis by Polygiannakis et al. (2002), in the interval 1994 2000 , indicates ephemeral periodicities of 27, 59, 137 and 330 days for flares and 37, 97, 182 and 365 days for CMEs.

Solar wind, being the continuation of solar corona, exhibits also periodic behaviour possibly be driven by the Sun periodic variations. The terrestrial magnetic field-solar wind interaction triggers magnetospheric activity (ionospheric electric currents, auroras, magnetic storms, turbulence and magnetic reconnection) quantified by the global magnetospheric indices. As the interaction depends on the solar wind physical characteristics, the indices are expected to exhibit similar periodicities as the solar wind. Therefore, the study of the periodicities of the solar wind and the geomagnetic indices lead to understanding the connection between them and the solar activity.

This work examines these joint solar wind and magnetospheric variations within the 1966-2010 interval which includes four solar cycles. The time series examined exhibit a non stationary, quasi-periodic behaviour where periodic components appear intermittently and with varying significance levels. We use, therefore, wavelet analysis in our study in addition to the classic Lomb/Scargle periodogram (Lomb, 1976; Scargle, 1982) which is used for comparison. 


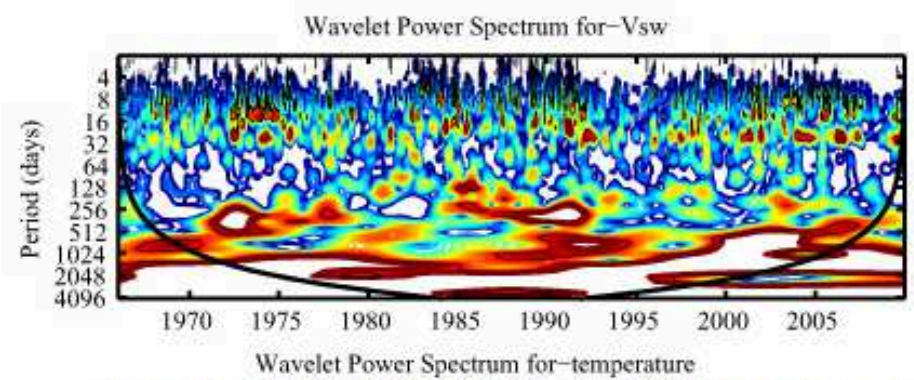

Global Wavelet Spectrum
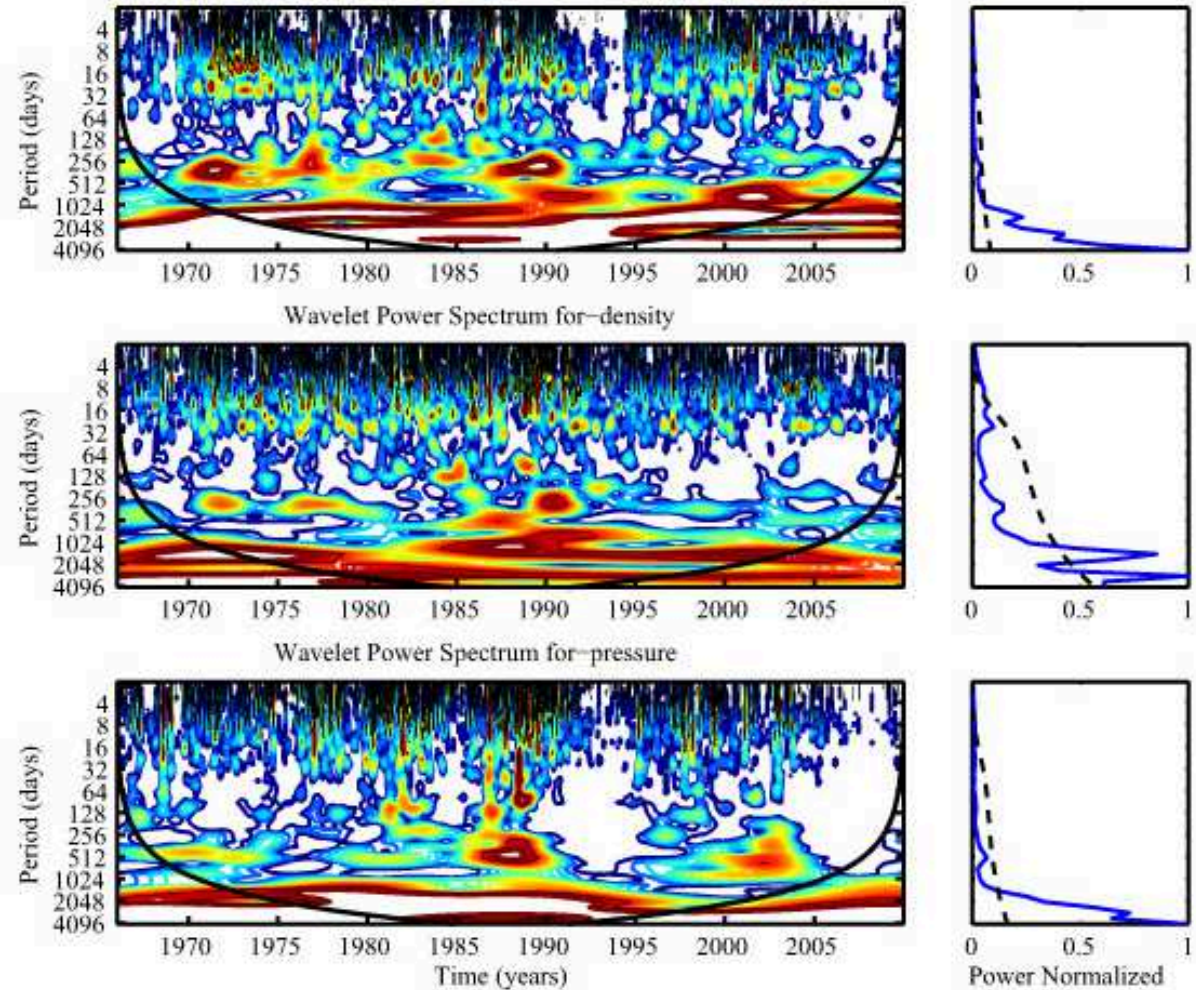

Figure 1. Wavelet spectra of solar wind parameters (Top to bottom): speed $\left(V_{s w}\right)$, plasma temperature $(\mathrm{T})$, plasma density $(\mathrm{D})$ and plasma pressure $(\mathrm{P})$. (Our plot is colour-coded with red corresponding to the maxima; the black contour is the $99 \%$ confidence level). The normalized global wavelet spectra (see 2.2) are shown on the right of each panel; in this case the dashed lines represent confidence level above $99 \%$.).

\section{Observations and Data Analysis}

\subsection{Data Selection}

The solar wind parameters examined are speed $\left(V_{s w}\right)$, proton density $(\mathrm{D})$, temperature $(\mathrm{T})$, plasma pressure $(\mathrm{P})$, inter-planetary magnetic field $\left(\mathrm{B}_{x}, \mathrm{~B}_{y}\right.$, and $\left.\mathrm{B}_{z}\right)$, Alfven Mach number $\left(M_{A}\right.$, local flow velocity to the local Alfven speed), and 


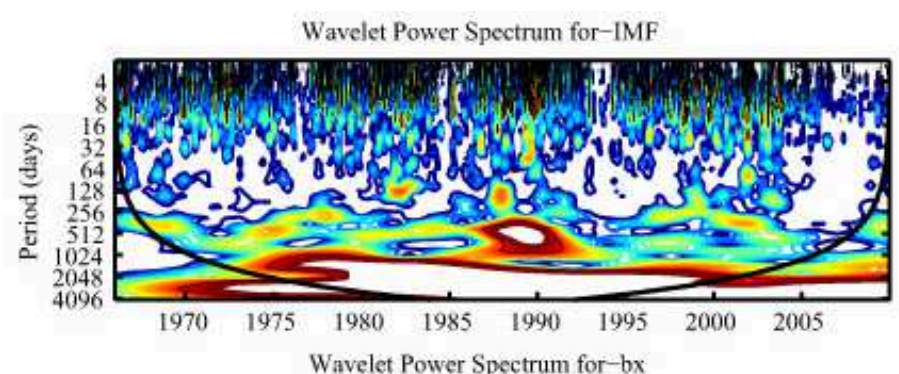

Global Wavelet Spectrum
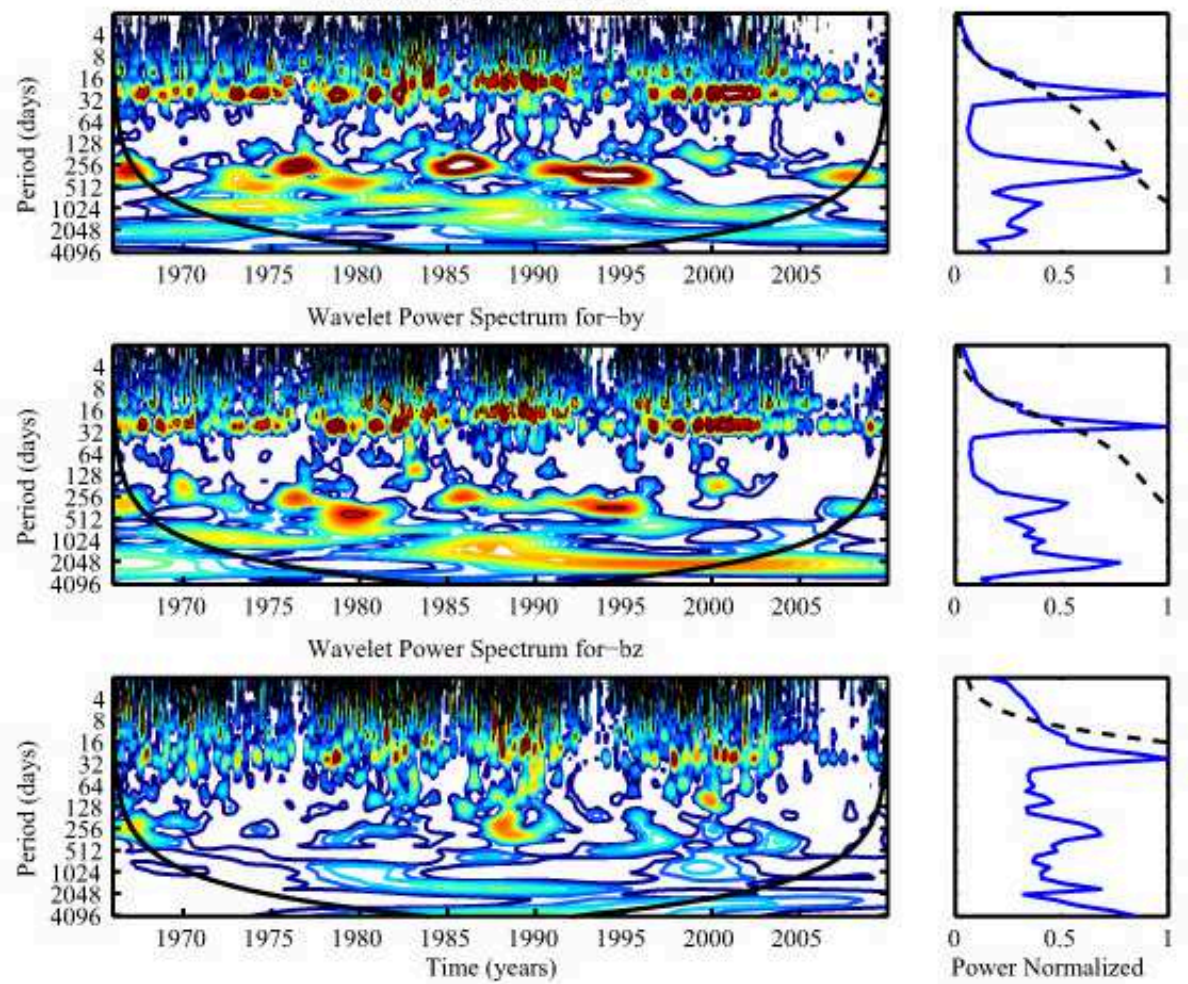

Figure 2. Same as Figure 1 for the interplanetary magnetic field (IMF) and its components $\mathrm{B}_{x}, \mathrm{~B}_{y}$ and $\mathrm{B}_{z}$.

plasma beta ( $\beta=n k T /\left[B^{2} / \mu_{0}\right]$, plasma pressure to the magnetic pressure ratio). The corresponding geomagnetic indices studied are, the $D_{S T}$ (a measure of the strength of Earth's ring current) 1 , the $K_{p}$ (mean value of the disturbance levels in the two geomagnetic horizontal field components), the $A_{p}$ (linear version of $K_{p}$ ) and Aurora Electrojet index AE. The latter is a global, quantitative measure of auroral zone magnetic activity produced by enhanced ionospheric currents flowing below and within the auroral oval.

${ }^{1}$ The $D_{S T}$ Index is calculated by averaging the horizontal component of the magnetic field from mid-latitude and equatorial magnetograms 

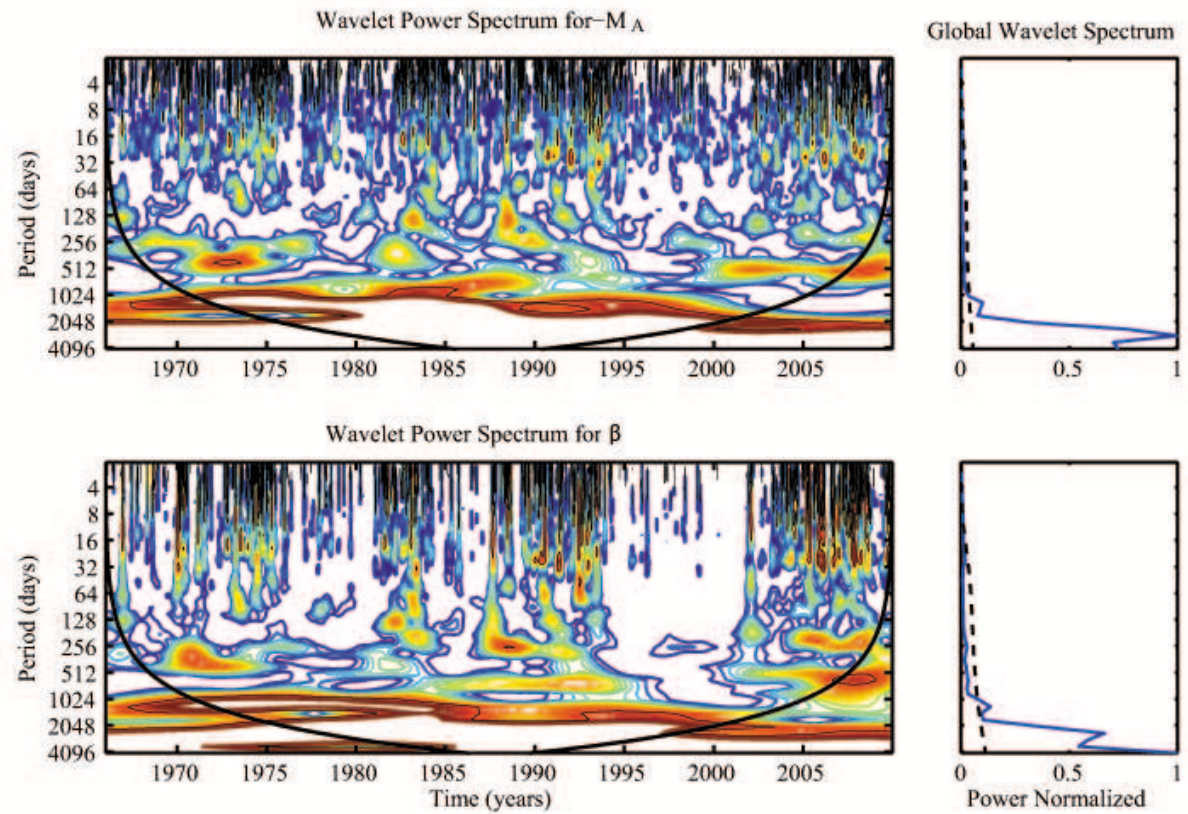

Figure 3. Same as Figures 1 and 2 Alfven Mach number $\left(M_{A}\right.$, Upper panel $)$ and plasma beta $(\beta$, lower Panel).

The OMNI database 2 was used and all data are measured at the distance of 1 A.U. in daily values from January 1st, 1966 to December 31st, 2010 (last four solar cycles).

\subsection{Wavelet analysis}

As we analyze non-stationary time-series we expand in terms of time-localized waves, or wavelets in order to obtain a compact, two dimentional, representation (see Morlet et al., 1982; Torrence and Compo, 1998) of these time-series:

$$
y\left(t, t^{\prime}, f\right)=\exp (2 i \pi f t) \exp \left(-f^{2} \frac{\left(t-t^{\prime}\right)^{2}}{2}\right)
$$

where $\mathrm{f}$ is the frequency, $\mathrm{t}$ ' the time delay and

$$
\exp \left(-f^{2} \frac{\left(t-t^{\prime}\right)^{2}}{2}\right)
$$

is the Gaussian support.

We use the Morlet wavelet because it's the most common wavelet function used for revealing periodicities of astrophysical signals; this makes easier the

${ }^{2}$ http://omniweb.gsfc.nasa.gov 


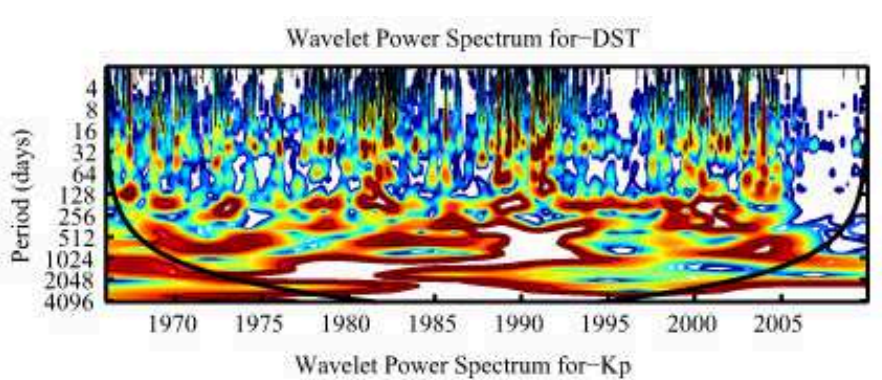

Global Wavelet Spectrum
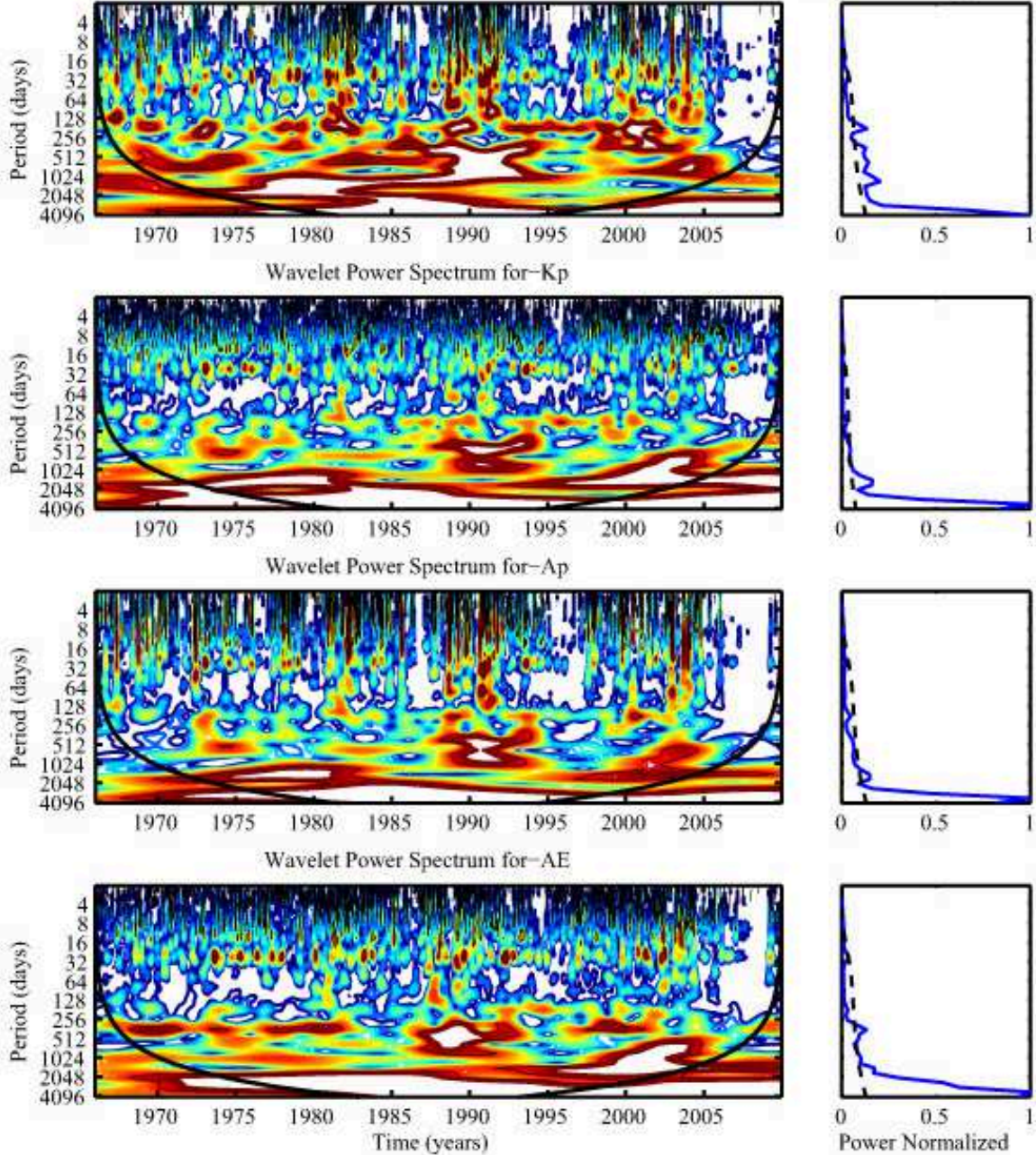

Figure 4. Same as Figures 12 and 3 for the geomagnetic indices (Top to bottom) ( $\mathrm{D}_{S T}, \mathrm{~K}_{p}$, $\mathrm{A}_{p}$ and $\left.\mathrm{AE}\right)$.

comparison of our results with previously published works. Furthermore, due to its Gaussian support, the Morlet wavelet expansion inherits optimality as regards the uncertainty principle (Morlet et al. 1 , 1982).

We also calculate the global wavelet spectrum (see Torrence and Compo, 1998) which is the time-averaged wavelet spectrum when the average is over all the local wavelet spectra and is given by the equation

$$
\bar{W}^{2}(s)=1 / N \sum_{n=0}^{N-1}\left|W_{n}(s)\right|^{2}
$$


Wavelet Analysis on Solar Wind Parameters and Geomagnetic Indices

Table 1. Periodicities (in days) indicated by Wavelet analysis. These periodicities span a range of periods; the corresponding peak is within parenthesis next to the range. The b-superscript marks periodicities which are below confidence level of $99 \%$ in the global wavelet spectrum. The column labeled interval presents the length of time (occasionally more than one) within which the range of Mid-term periodicitities was detected; as similar column was omitted from the long-term periodicities as in this case the interval was always equal to 44 years.

\begin{tabular}{|c|c|c|c|c|}
\hline Par. & Short-term & Mid-term & Interval & Long-term \\
\hline $\mathrm{V}_{S W}$ & $\begin{array}{l}9-14(13.9) \\
15-25^{b} \\
22-30(27.8)\end{array}$ & $\begin{array}{l}256-512(314) \\
512-1024(629)\end{array}$ & $\begin{array}{l}7-8 \text { years } \\
4-11 \text { years }\end{array}$ & $1024-4096(1499,2998)$ \\
\hline Temp. & $\begin{array}{l}9-22(13.9) \\
15-25^{b} \\
22-30^{b}(27.8)\end{array}$ & $\begin{array}{l}256-445^{b}(314) \\
512-1024^{b}(889)\end{array}$ & $\begin{array}{l}3-4 \text { years } \\
6 \text { years }\end{array}$ & $1024-4096(1499,2521)$ \\
\hline Density & $\begin{array}{l}9-14^{b} \\
15-25^{b}\end{array}$ & $\begin{array}{l}256-445^{b}(314) \\
512-1024^{b}\end{array}$ & $\begin{array}{l}3-4 \text { years } \\
10 \text { years }\end{array}$ & $1024-4096(1499,2998)$ \\
\hline Pressure & & $256-630(528)$ & 4 years & $1024-4096(2998)$ \\
\hline IMF B & & $256-1024(444)$ & 6 years & $1024-4096(3565)$ \\
\hline $\mathrm{B}_{x}$ & $\begin{array}{l}9-22(13.9) \\
22-30(27.8)\end{array}$ & $256-512(314)$ & $3-6$ years & \\
\hline $\mathrm{B}_{y}$ & $\begin{array}{l}9-22(13.9) \\
22-30(27.8)\end{array}$ & $256-512^{b}(314)$ & $3-4$ years & \\
\hline $\mathrm{B}_{z}$ & $\begin{array}{l}\left.9-22^{b} 13.9\right) \\
22-30^{b} \quad(27.8)\end{array}$ & & & \\
\hline $\mathrm{M}_{A}$ & & $128-512^{b}$ & 4-10 years & $1024-4096(1260,2998)$ \\
\hline$\beta$ & $14-30^{b}(27.8)$ & $128-512^{b}$ & 5 years & $1024-4096(1260,2521)$ \\
\hline $\mathrm{D}_{S T}$ & $\begin{array}{l}9-22^{b}(13.9) \\
22-30(27.8) \\
64-128\end{array}$ & $\begin{array}{l}128-256(187) \\
256-512(374) \\
512-1024(629)\end{array}$ & $\begin{array}{l}2-11 \text { years } \\
6-15 \text { years } \\
7-15 \text { years }\end{array}$ & $1024-4096(1260,4096)$ \\
\hline $\mathrm{K}_{p}$ & $22-30^{b}(27.8)$ & $\begin{array}{l}128-256^{b}(187) \\
256-1024(374)\end{array}$ & $\begin{array}{l}1-5 \text { years } \\
4-6 \text { years }\end{array}$ & $1024-4096(1499,3565)$ \\
\hline $\mathrm{A}_{p}$ & $\begin{array}{l}12-30^{b}(13.9,27.8) \\
64-128^{b}\end{array}$ & $\begin{array}{l}128-256^{b}(187) \\
256-1024^{b}(374)\end{array}$ & $\begin{array}{l}1-2 \text { years } \\
3-6 \text { years }\end{array}$ & $1024-4096(1260,3565)$ \\
\hline $\mathrm{AE}$ & $22-30^{b} \quad(27.8)$ & $256-1024^{b}(374)$ & $5-8$ years & $1024-4096(1499,3565)$ \\
\hline
\end{tabular}

where $W_{n}(s)$ is the wavelet power and $\mathrm{N}$ the number of local wavelet spectra. Thus we obtain an unbiased and consistent estimation of the true power spectrum of a time series. 

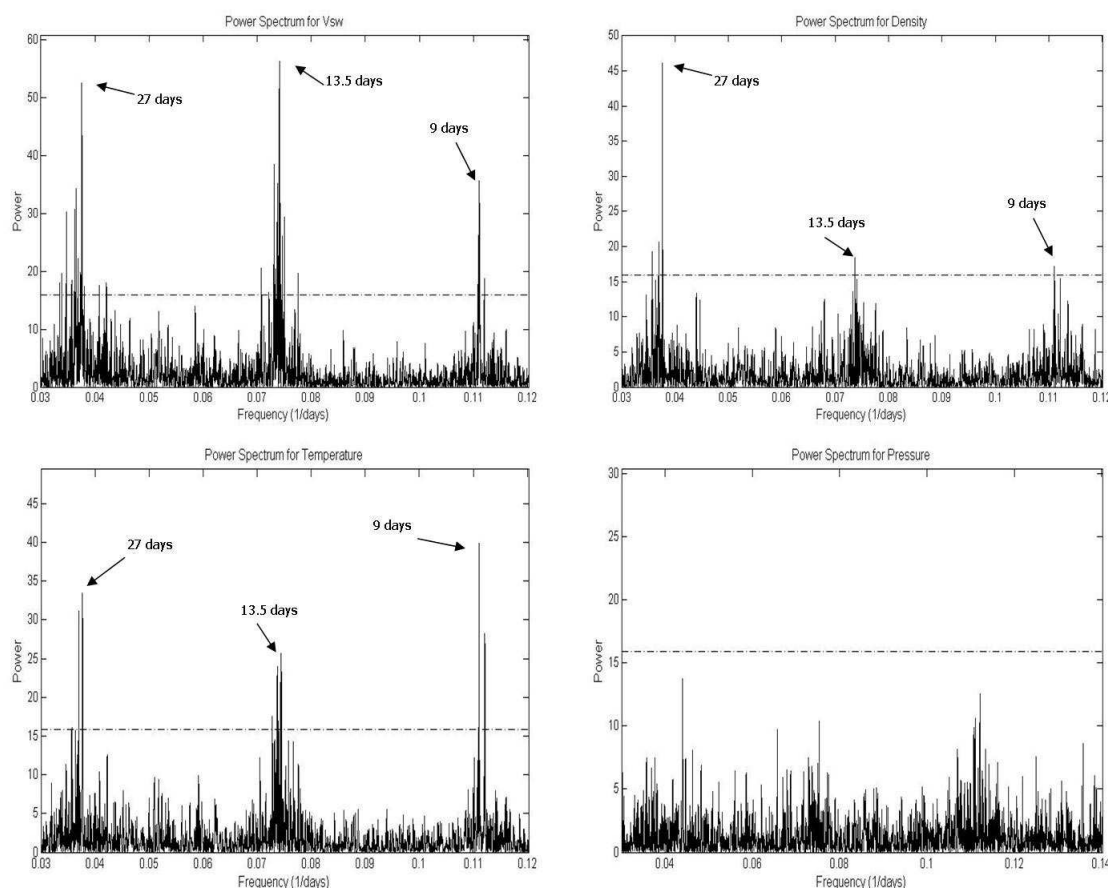

Figure 5. Lomb/Scargle periodogram for the short-term periodicities of the four dynamic parameters of the solar plasma: solar wind speed (Vsw), plasma temperature (T), plasma density (D) and plasma pressure $(\mathrm{P})$. (The dashed line in all plots denotes the $99 \%$ confidence level - peaks below this line are considered insignificant.)

2.3. Wavelet spectra of solar wind parameters and geomagnetic indices

In this section we examine the variation of the parameters of the solar wind and the geomagnetic indices; these provide us with fourteen time series in total. We use the wavelet power spectra and the global wavelet spectra for the identification of periodic components to a confidence leve 3 of $99 \%$.

Starting from the periodicities of the solar wind speed, temperature, density and pressure (figure 10 we note certain similarities as follows:

- The long-term periodicities (1024-4096 days) appear throughout the observation period regarding speed, temperature, density (with peaks at 4.1 and 8.2 years) and pressure (peak at 8.2 years only).

- Mid-term periodicities (256-512 days with peak at 314 days) in speed appear in 1970-1977 and 1985-1992. These appear also in temperature and density (peak 314 days). Periodicities of 512-1024 days appear in speed (peak 1.7 years) at the rising phase and maximum of cycle 20 and at cycles 22 and 23 and (below confidence level) in temperature (peak 2.4 years) at cycles 22 and 23 and pressure (peak 528 days).

${ }^{3}$ The confidence interval is defined as the probability that the true wavelet power at a certain time and scale lies within a certain interval about the estimated wavelet power. 

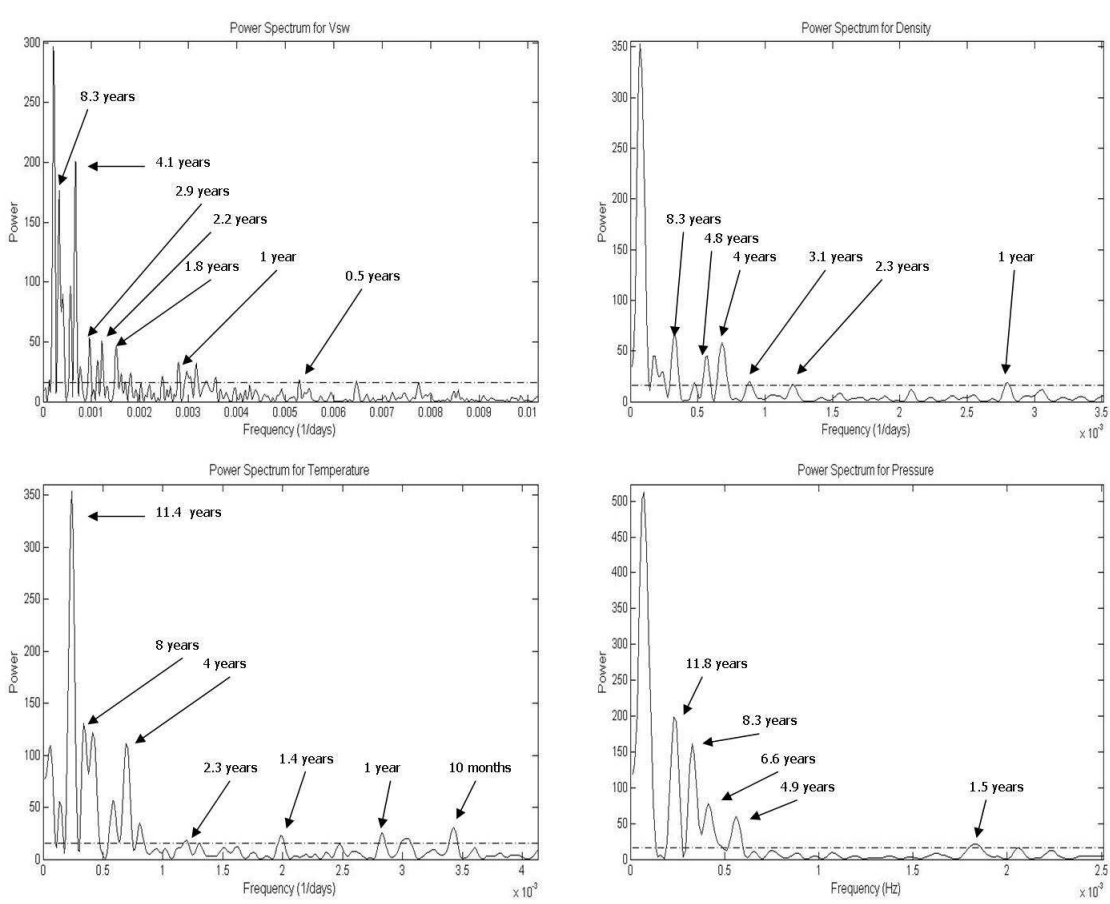

Figure 6. Lomb/Scargle periodogram for the long-term periodicities of the four dynamic parameters of the solar plasma: solar wind speed $\left(\mathrm{V}_{s w}\right)$, plasma temperature $(\mathrm{T})$, plasma density (D) and plasma pressure (P). (The dashed line in all plots denotes the $99 \%$ confidence level - peaks below this line are considered insignificant.)

- Short-term periodicities of 9-14 (peak 13.9) and 22-30 (peak 27.8) days, corresponding to the rotation of the sun and sub-harmonic, appear quite pronounced in the speed during the descending phases of cycles 20 and 23 but less pronounced during cycles 21, 22. These short-term periodicities appear in temperature and density as well but below the $99 \%$ confidence level.

Next, we isolate the periodic components of the interplanetary magnetic field examining $\mathrm{B}, \mathrm{B}_{x}, \mathrm{~B}_{y}, \mathrm{~B}_{z}$, Alfvén Mach number and plasma beta (Figures 2 and 3):

- Long-term periodicities of (1024-4096 days) days appear throughout the observation period in IMF B (peak 9.8 years), Alfvén Mach number (peaks 3.5 and 8.2 years) and plasma beta (peaks 3.5 and 6.9 years).

- Mid-term periodicities (256-1024 days with peak at 1.2 years) appear around the maximum of cycle 22 in IMF B. A range of 256-512 days (peak 314 days) periodicities appear in $\mathrm{B}_{x}$ (and $\mathrm{B}_{y}$ below the confidence level) during the ascending phases of cycles 20,21 and 22 and the descending phase of 22. The mid-term periodicities in Alfvén Mach number and plasma beta are below the confidence level. 

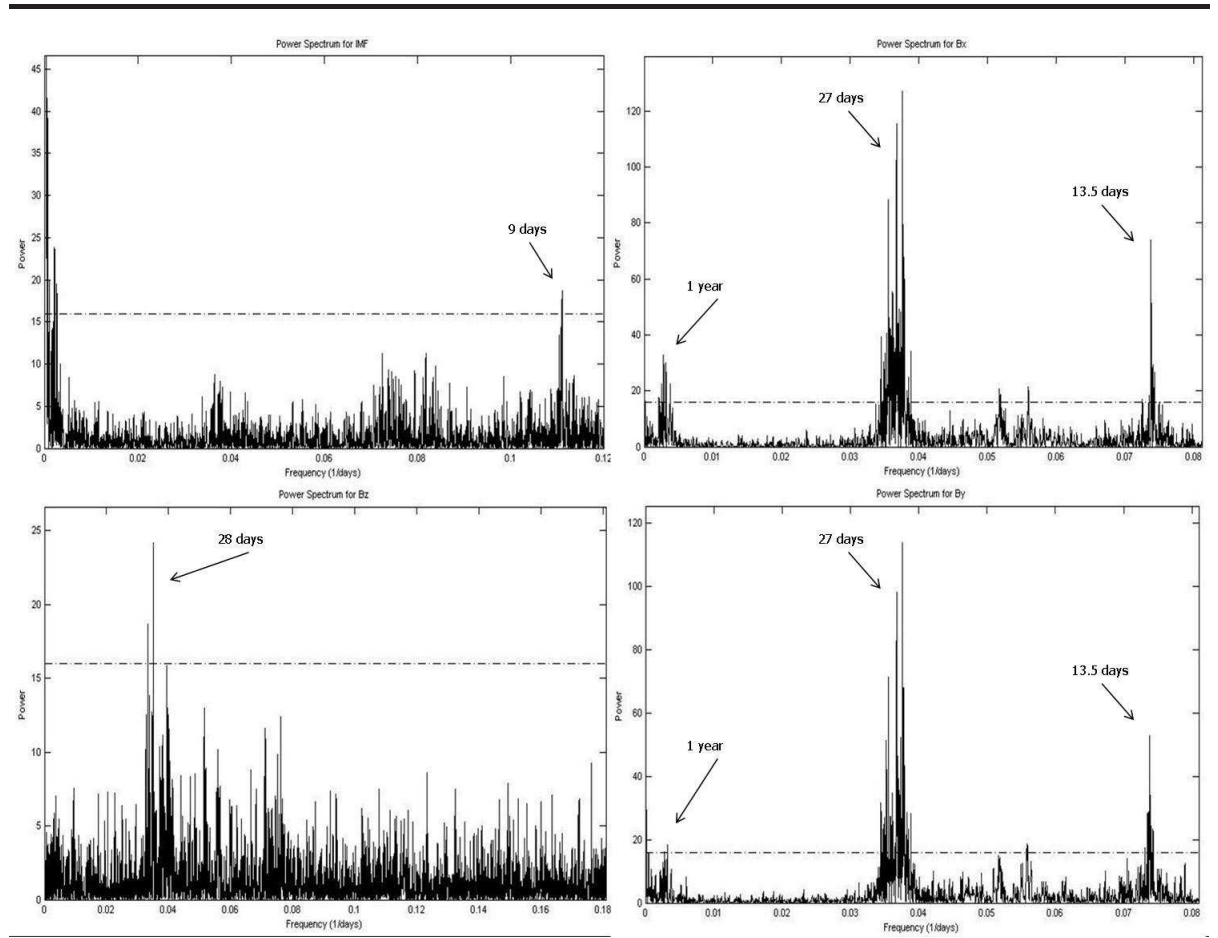

Figure 7. Lomb/Scargle periodogram of the interplanetary magnetic field (IMF) and its components $\mathrm{x}, \mathrm{y}, \mathrm{z}$. (The dashed line in all plots denotes the $99 \%$ confidence level - peaks below this line are considered insignificant.)

- Short-term periodicities of 9-22 days (peak 13.9 days) and 22-30 days (peak 27.8 days) appear in $\mathrm{B}_{x}$ and $\mathrm{B}_{y}\left(\mathrm{~B}_{z}\right.$ and plasma beta below the confidence level) throughout the observation period except the minima of cycle 22 and 23 around 1995 and 2005.

Finally we examine the periodicities of geomagnetic indices $\mathrm{D}_{S T}, \mathrm{~K}_{p}, \mathrm{~A}_{p}$ and AE (Figure 4):

- Long-term periodicities of 1024-4096 days appear throughout the whole observation period in $\mathrm{D}_{S T}$ (peaks 3.5 and 10.8 years), $\mathrm{K}_{p}$ (peaks 4.1 and 9.8 years), $\mathrm{A}_{p}$ (peaks 3.5 and 9.8 years) and $\mathrm{AE}$ (peaks 4.1 and 9.8 years).

- Mid-term periodicities in geomagnetic indices vary: A periodicity range of 256-1024 days (peak 1 year) in $\mathrm{AE}, \mathrm{K}_{p}$ and $\mathrm{A}_{p}$ (below confidence level) appears during the maximum and descending phase of cycle 22 . The same extends in $\mathrm{D}_{S T}$ (with peaks 1 year and 1.7 years) but in the time intervals 1969-1975, 1980-1995 and 2000-2005.

- A smaller range of periodicities of 512 - 1024 days appear in $\mathrm{K}_{p}, \mathrm{~A}_{p}$ and $\mathrm{AE}$ in 2000-2005.

- A smaller range of periodicities of 256-512 days (peak 1 year) appear around the maximum of every cycle in AE. 

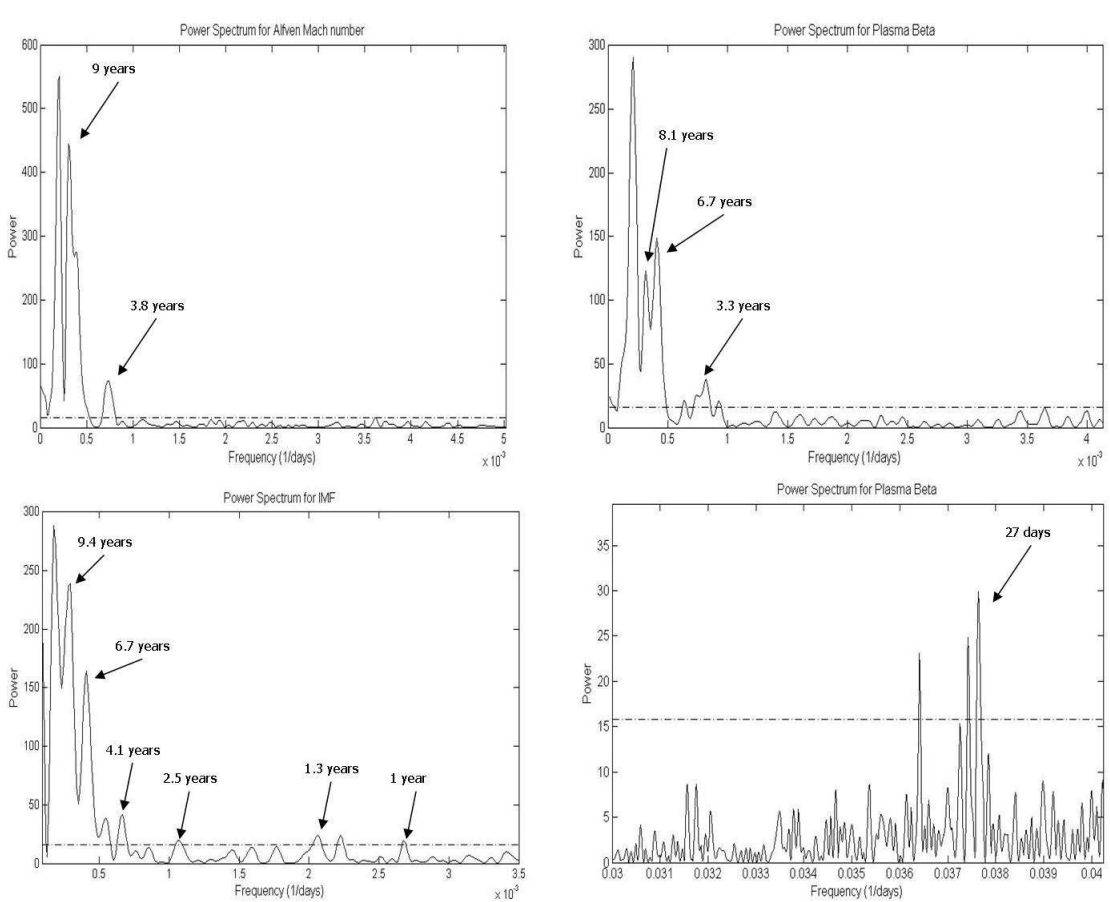

Figure 8. Lomb/Scargle periodogram for the long-term periodicities of the interplanetary magnetic field (IMF), Alfven Mach number and plasma Beta and the short-term periodicities of Plasma Beta. (The dashed line in all plots denotes the $99 \%$ confidence level - peaks below this line are considered insignificant.)

- Short-term periodicities (22-30 days, with 27.8 days peak) appear intermittently and without any obvious pattern in all four indices (above the confidence level only $\left.\mathrm{D}_{S T}\right)$. Periodicities of $64-128$ days are observed in $\mathrm{D}_{S T}$ (maxima of cycles 21, 22, 23 and descending phase of 23) and $\mathrm{A}_{p}$ (maximum of cycle 22 and descending phase of 23 below the confidence level in both cases). Also periodicities of 128-256 days (peak 187 days) appear in $\mathrm{D}_{S T}$ throughout the observation period up to 2004, these periodicities extend to $\mathrm{K}_{p}$ and $\mathrm{A}_{p}$ but below the confidence level.

\subsection{Lomb/Scargle method}

Scargle (1982) modified the standard periodogram formula to first find a time delay $\tau$ such that the power of each frequency was indepedent of any constant shift of sample time $t_{j}$ and equivalent to the reduction of the sum of squares in least-squares fitting of sine waves to the data. The time delay $\tau$ is defined as:

$$
\tan (2 \omega \tau)=\frac{\sum_{j} \sin \left(2 \omega t_{j}\right)}{\sum_{j} \cos \left(2 \omega t_{j}\right)}
$$



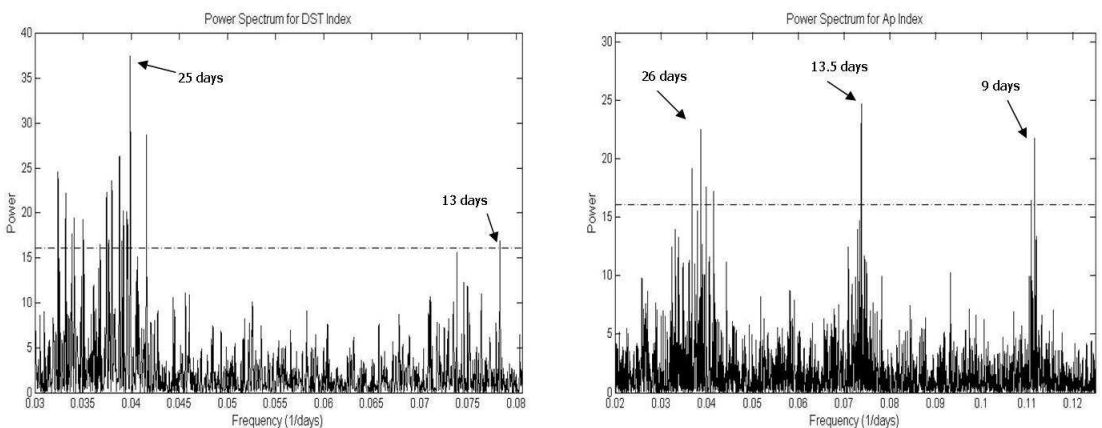

Power Soectum for AE Inder
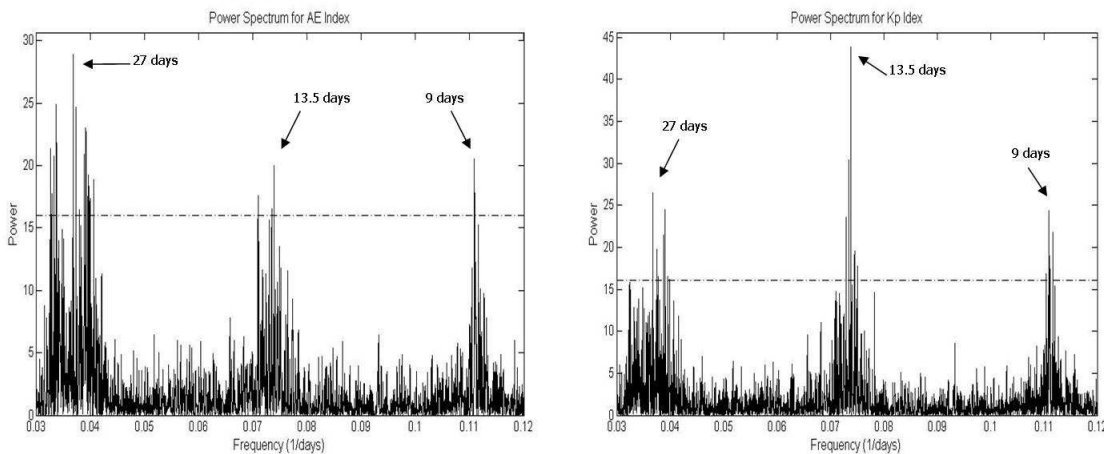

Figure 9. Lomb/Scargle periodogram for the short-term periodicities of the geomagnetic indices $\left(\mathrm{D}_{S T}, \mathrm{AE}, \mathrm{K}_{p}\right.$ and $\mathrm{A}_{p}$ ). (The dashed line in all plots denotes the $99 \%$ confidence level - peaks below this line are considered insignificant.

The periodogram at frequency $\omega$ is then estimated as:

$$
P_{x}(\omega)=1 / 2 \frac{\left[\sum_{j} X_{j} \cos \left(t_{j}-\tau\right)\right]^{2}}{\sum_{j} \cos 2\left(t_{j}-\tau\right)}+1 / 2 \frac{\left[\sum_{j} X_{j} \sin \left(t_{j}-\tau\right)\right]^{2}}{\sum_{j} \sin 2\left(t_{j}-\tau\right)}
$$

where $\omega$ is the frequency and $\mathrm{X}_{j}$ is the value of the physical quantity measured in time $t_{j}$, The Lomb/Scargle periodogram, being a variant of the Fourier transform with application in unevently spaced data, represents a signal as the sum of sine and cosine functions of infinite duration. More often than not, however, the signal statistical properties, characteristic periodicities included, vary over time so this method provides information about the frequency content of a time series without localizing it in time. The discrete periodicities in the Lomb/Scargle periodogram sometimes correspond to a range of periodicities in the wavelet spectrum.

2.5. Lomb/Scargle periodogram of solar wind parameters and geomagnetic indices

In this section we present the Lomb/Scargle periodogram for each parameter of the solar wind and geomagnetic index (figures 5 to 10). The periodicities we accept are those above the confidence level of $99 \%$. 


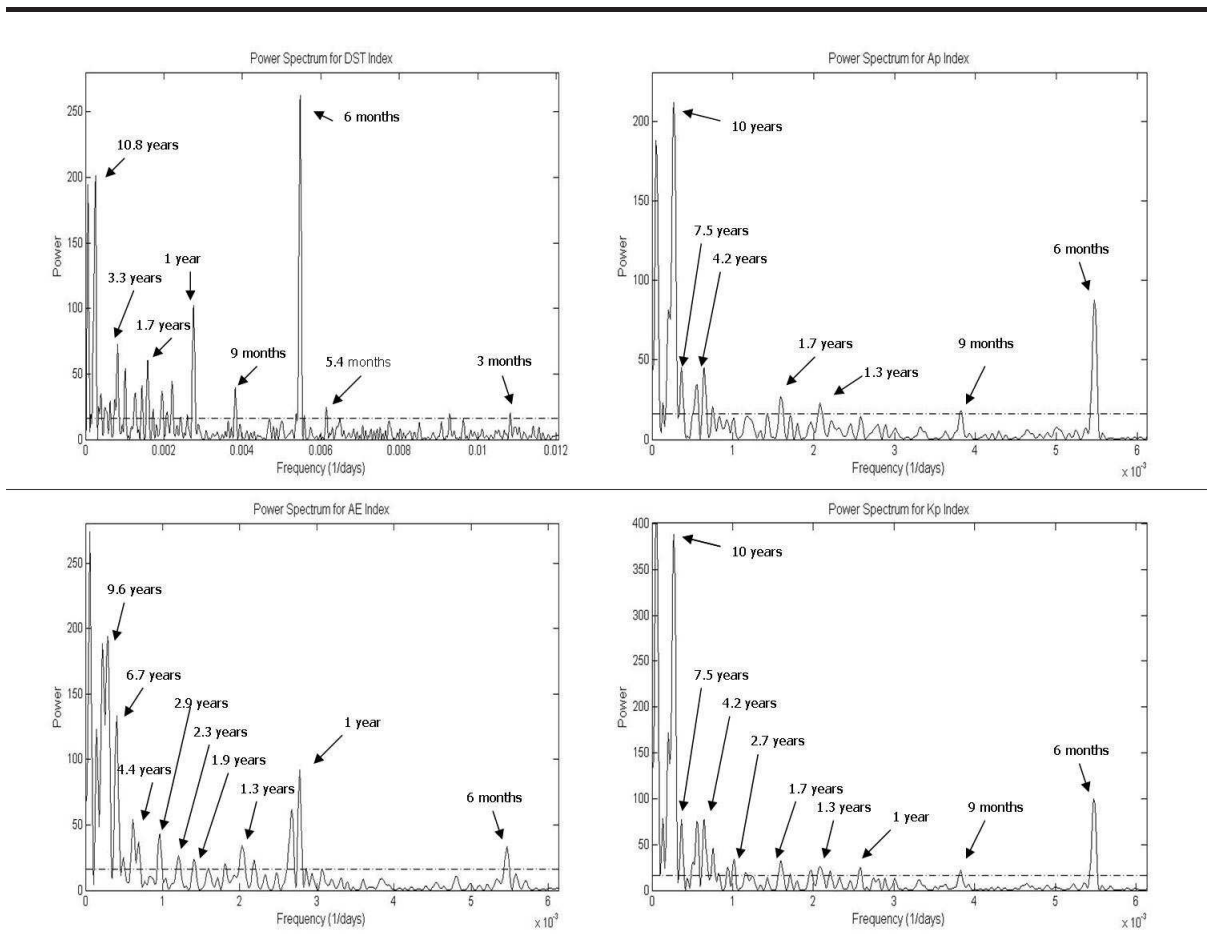

Figure 10. Lomb/Scargle periodogram for the long-term periodicities of the geomagnetic indices $\left(\mathrm{D}_{S T}, \mathrm{AE}, \mathrm{K}_{p}\right.$ and $\mathrm{A}_{p}$ ). (The dashed line in all plots denotes the $99 \%$ confidence level - peaks below this line are considered insignificant.)

In figure 5 and 6 we present periodicities of the four parameters of the solar wind (speed, temperature, density and pressure). There are similarities at speed, density and temperature as stated also in section 2.2 .

- The short-term periodicities of $9,13.5$ and 27 days appear in all three parameters (figure 5).

- The annual periodicity appears in all four parameters (figure 6) but with slight differences (316 and 358 days in speed, 329 and 353 in temperature, 357 in density and 316 and 357 in pressure). There is also a semi-annual periodicity only in speed.

- There are periodicities of 554 ad 657 days in speed, 502 days in temperature and 544 days in pressure.

- A 2.2-2.3 years periodicity (823, 834 and 830 days) in speed, temperature and density respectively is noted.

- Long-term periodicities appear in all four parameters with maxima at 2.9, 4.1 and 8.3 years for speed, 4,8 and 11.4 years for temperature, 3.1, 4, 4.8 and 8.3 years for density and $4.9,6.6,8.3$ and 11.8 years for pressure.

In figures 7 and 8 we present the interplanetary magnetic field, Alfvén Mach number and plasma beta. 
Katsavrias et al.

Table 2. Table of periodicities (in days) revealed by the Lomb/Scargle periodogram. The confidence level is above $99 \%$.

\begin{tabular}{|c|c|c|c|}
\hline Parameter & Short-term & Mid-term & Long-term \\
\hline $\mathrm{V}_{S W}$ & $9,13.5,27$ & $182,316,358,554,657,823$ & $1032,1513,3026$ \\
\hline Temperature & $9,13.5,27$ & $291,329,502,353,834$ & $1441,2937,4173$ \\
\hline Density & $9,13.5,27$ & 357,830 & $1126,1468,1749,3045$ \\
\hline Pressure & & $316,357,544$ & $1788,2403,3045,4383$ \\
\hline IMF B & 9 & $373,485,934$ & $1507,1827,2458,3446$ \\
\hline $\mathrm{B}_{x}$ & 13.527 & 353 & \\
\hline $\mathrm{B}_{y}$ & 13.527 & 313,353 & \\
\hline $\mathrm{B}_{z}$ & 2830 & & \\
\hline $\mathrm{M}_{A}$ & & & 1376,3268 \\
\hline$\beta$ & 27 & & $1220,2459,2956$ \\
\hline \multirow[t]{2}{*}{$\mathrm{D}_{S T}$} & 13,25 & 182,261 & 1204 \\
\hline & 92 & 362,6632 & 3942 \\
\hline \multirow{3}{*}{$\mathrm{K}_{p}$} & 9 & 182,262 & 1541 \\
\hline & 13.5 & 388,81 & 2739 \\
\hline & 27 & 986,624 & 3652 \\
\hline \multirow{3}{*}{$\mathrm{A}_{p}$} & 9 & 182 & 1541 \\
\hline & 13.5 & 261 & 2739 \\
\hline & 26 & 481,628 & 3652 \\
\hline \multirow{3}{*}{$\mathrm{AE}$} & 9 & 183,360 & 1055,1601 \\
\hline & 13.5 & 492,711 & 2441 \\
\hline & 27 & 828 & 3503 \\
\hline
\end{tabular}

- There is an identical behaviour of the $\mathrm{x}$ and $\mathrm{y}$ component of the IMF where we have the appearance of three periodicities of 13.5 and 27 days and 1 year.

- IMF appears a periodicity of 9 days (figure 7) and mainly long-term periodicities of $1,1.3,2.5,4.1,5,6.7$ and 9.4 years (figure 8).

- The z component seems to appear only a 29-30 days periodicity.

- Long term periodicities are mainly appearing in Alfvén Mach number (figure 8) with peaks of 3.8 and 9 years.

- Plasma beta appears a periodicity of 3.3,6.7 and 8.1 years and also the 27 days periodicity.

The Lomb/Scargle periodograms in geomagnetic indices are presented in figures 9 and 10

- There are once again similarities in $\mathrm{K}_{p}, \mathrm{~A}_{p}$ index. Short-term periodicities (figure 9) of 9, 13.5 and 27 days appear in $\mathrm{K}_{p}, \mathrm{~A}_{p}$ and $\mathrm{AE}$, while $\mathrm{D}_{S T}$ shows periodicities of 13 days and 25 days. 
- Periodicities of 0.5 and 0.7 years (figure 10) appear in $\mathrm{K}_{p}$ and $\mathrm{A}_{p}$ index as well as in $\mathrm{D}_{S T}$, while $\mathrm{AE}$ contains only the 6 month periodicity. The $\mathrm{D}_{S T}$ shows also a 92 days periodicity.

- Long-term periodicities of 1-2.8 years appear in all indices but with different power and maxima. Maxima of 1.3, 1.7, 4.2, 7.5 and 10 years in $\mathrm{A}_{p}$ and 1.3, 1.7, 2.7, 4.2 7.5 and 10 in $\mathrm{K}_{p}$ are the most important, while AE index shows peaks of $1,1.3,1.9,2.3,2.9,4.4,6.7$ and 9.6 years.

- $\mathrm{D}_{S T}$ shows $1,1.7,3.3$ and 10.8 years maxima.

\section{Summary and discussion}

A number of short and long-term periodicities were detected within the $99 \%$ confidence level. We, firstly, draw attention to similarities in periodic behaviour between certain parameters of the solar wind and the magnetosphere.

1. Solar wind speed and temperature are expected to be correlated (see the study Katsavrias. Preka-Papadema, and Moussas, 2010, for cycle 23); they share the same periodicities in our data.

2. There is symmetrical behavior of the $\mathrm{x}$ and $\mathrm{y}$ component $\left(\mathrm{B}_{x}, \mathrm{~B}_{y}\right)$ of the IMF, so they exhibit identical periodicities

3. As $\beta \approx M_{A}^{2}$ they, too, share the same periodicities.

4. Similar behaviour is also expected from $\mathrm{K}_{p}$ and $\mathrm{A}_{p}$ index since the latter is a linear version of the former.

\subsection{Short-term periodicities}

These short-term periodicities, due to the solar rotation, were detected in solar wind $\left(\mathrm{V}_{S W}\right)$ speed with enhanced amplitude during the descending phases of cycles 20 and 23 but less pronounced during cycles 21, 22; the latter cycles had larger maxima and shorter minima. Short-term periodicities appeared in temperature and density as well (but below the accepted $99 \%$ confidence level) outside peaks of cycles. Prabhakaran Navar et al. (2002) observed these periodicities of $\mathrm{V}_{S W}$ in the descending phases of 21, 22 and 23, while José Alves Bolzan et al. (2005), report similar findings in speed and density in the years 1997 (less pronounced) and 2000. Periodicities of about 27.5, 13.5, 9.1, and 6.8 days were identified in the solar wind speed for the time interval 1964-2000 (Gonzalez and Gonzalez, 1987: Clúa de Gonzalez et al., 1993; Svalgaard and Wilcox, 1975; Fenimore et al., 1978). We found the 9, 13.5 and 27 days periodicities using the Lomb/Scargle periodogram. It's been proposed that intervals of large 13.5-day periodicity are due to the occurrence at $1 \mathrm{AU}$ of two high-speed streams per solar rotation (Mursula and Zieger, 1996).

In $\mathrm{V}_{S W}$ (and $\mathrm{T}$ below the $99 \%$ confidence level) the 27 days periodicity is quite apparent during all four solar cycles, covered in our date set, apart from each cycle's maximum. This is probably due to the fact that fast streams from coronal holes overlaps with mass ejection from or near the active regions resulting in a, more or less, random variation of the measured solar wind speed. In support of this line of reasoning, we note, that in cycles 21 and 22 where the maxima 
have benn quite pronounced and the minima of short duration, this effect of the suppression of the spectral peak in $\mathrm{V}_{S W}$ was more evident compared to cycles 20 and 23.

Periodicities of 27.5, 13.5, 9.1, and 6.8 days were identified in the IMF polarity for the time intervals 1964-2000 (Gonzalez and Gonzalez, 1987; Clúa de Gonzalez et al., 1993; Svalgaard and Wilcox, 1975; Fenimore et al., 1978; Mursula and Zieger, 1996; Prabhakaran Nayar et al., 2002). We found short-term periodicities of 9-22 days (peak 13.9 days) and 22-30 days (peak 27.8 days) appear in Bx throughout the observation period except the descending phases of cycle 22 and 23. These appear also in By and Bz but below confidence level. Lomb/Scargle periodogram showed the same periodicities.

\subsection{Annual and semi-annual periodicity}

CMEs show an annual and semi-annual periodicity during the time interval 1994-2000 (Polygiannakis et al., 2002) and an annual, semi-annual and 3 years periodicity during 1999-2003 (Lou et al., 2003). We found the annual periodicity in the Lomb/Scargle periodogram of speed, density, temperature, pressure, IMF, $\mathrm{B}_{x}$ and $\mathrm{B}_{y}$. Moreover, a semi-annual periodicity was found in speed and a 3 years periodicity in speed and density. This could be a strong argument for the dependence of the solar wind speed on CMEs but wavelet spectrum of solar wind show these periodicities ( 0.5 and 1 year) during solar cycles 20,21, 22 and not the 23rd. In addition, the annual periodicity appear with enhanced amplitude in the cycles 20 and 22 in speed, density and temperature.

According to Lou et al. (2003), $A_{p}$ index shows also periodicities of 187, 273 and 364 days during the time interval 1999-2003. We found a 182 days variation in $\mathrm{D}_{S T}$ throughout the whole observation period which in the other indices is below the confidence level. The 273 and 364 days periodicities are included in the range of 256-1024 days (peak 1 year) in $\mathrm{K}_{p}$ and (below confidence level) $\mathrm{A}_{p}$ during the maximum and descending phase of cycle 22 and in AE during the whole observation period except the minima. This indicates that CMEs are correlated with the geomagnetic storm disturbances via solar wind speed. The Lomb/Scargle periodogram shows a peak of 182 days in all four indices and a peak of 262 days ( 0.7 years) in $\mathrm{D}_{S T}, \mathrm{~K}_{p}$ and $\mathrm{A}_{p}$.

\subsection{1-2 years intermittent periodicity}

According to Obridko and Shelting (2007) the IMF shows a 1.3 years periodicity . We found this periodicity but only around the maximum of the cycle 22 which is probably been propagating from the solar magnetic field (SMF), with no obvious explanation about its absence from the other cycles.

As reported by Gazis, Richardson, and Paularena (1995) and Valdés-Galicia, Pérez-Enríquez, and Otaola (1996) solar wind speed, CMEs and galactic cosmic rays show intermittent and somewhat varying (1-1.3 years) periodicity during 1974-2000 and 1964-2000 respectively. Kudela et al. (2010) report that a range of periodicities 1.7-2.2 years, appear in Cosmic Rays during the time interval 1951-2010 and Mavromichalaki et al. (2003) during 1953-1996. The same periodic modulation has been detected when 
fluxes of solar energetic protons and galactic cosmic rays are investigated Laurenza et al. (2009). We found periodicities of 512-1024 days in speed (peak 1.7 years) at the ascending phase and maximum of cycle 20 and at cycles 22 and 23 , as well as below the confidence level - in temperature (maximum and descending phase of 22 and 23)and density (maximum and descending phase of 22). This is supported by the Lomb/Scargle periodogram that shows peaks of 1.5 and 1.8 years in speed and 1.4 years in temperature. It seems that there is a periodicity varing between $1.2-1.3$ years and a periodicity of 2 years varing between $1.7-2.2$ years.

Valdés-Galicia, Pérez-Enríquez, and Otaola (1996); Mursula (1999); Prabhakaran Nayar et al. (2002) report different periodic variations of the geomagnetic activity index $\mathrm{A}_{p}$; 1.3-1.4 years during even cycles and of 1.5-1.7 years during odd. The $\mathrm{A}_{p}$ (and $\mathrm{AE}$ below the confidence level) periodicities measured were within the 1.3-1.7 years range yet no distinction between even or odd cycles was apparent. On the other hand we found two intervals of periodic variations in all four indices $\left(\mathrm{D}_{S T}\right.$, $\mathrm{K}_{p}, \mathrm{AE}$ and $\mathrm{A}_{p}$ ), as follows:

1. In the 256-1024 days range from 1990 to 1995; these coincide with variations, at the same frequency range within the same interval, of the $\mathrm{V}_{S W}$, IMF (and $\mathrm{T}$ below the $99 \%$ confidence level).

2. 512-1024 days in 2000-2005; these coincide with variations, at the same frequency range within the same interval, of the $\mathrm{V}_{S W}$ (and $\mathrm{T}$ below the $99 \%$ confidence level) but not IMF this time.

We interprete this association as the result of the Solar Wind and IMF triggering and modulating magnetospheric activity. The effects seem to spread to all latitudes $\left(\mathrm{K}_{p}, \mathrm{AE}\right)$ and to the ring current as well $\left(\mathrm{D}_{S T}\right)$.

The Lomb/Scargle periodogram confirmes the $1.7-1.8$ years periodicity in $\mathrm{V}_{S W}, \mathrm{D}_{S T}, \mathrm{~K}_{p}, \mathrm{~A}_{p}$ and $\mathrm{AE}$ (below the $99 \%$ confidence level); this corroborates similar results by Valdés-Galicia. Pérez-Enríquez. and Otaola (1996).

\subsection{Long-term periodicities}

Periodicities of 1024-4096 days appear throughout the whole observation period in solar wind speed (peaks 4.1 and 8.2 years), temperature (peaks 4.1 and 6.9 years), density (peaks 4.1 and 8.2 years) and pressure (peak 8.2 years). These also extend to $\mathrm{D}_{S T}$ (peaks 3.5 and 11.2 years), $\mathrm{K}_{p}$ (peaks 4.1 and 9.8 years), Ap (peaks 3.5 and 9.8 years) and AE (peaks 4.1 and 9.8 years). The corresponding Lomb/Scargle periodogram gives us also peaks of 2739 days (7.7 years) and 1204 days (3.3) in $\mathrm{K}_{p}$ and $\mathrm{A}_{p}$. The former was also found in cosmic rays in 1953-1996 (Mavromichalaki et al., 2003) and the latter in 1947-1990 (Valdés-Galicia, Pérez-Enríquez, and Otaola, 1996) .

A 9.8 years periodicity was also found throughout the four cycles corroborating findings of Prabhakaran Navar et al. (2002) in 1964-2000. In the Lomb/Scargle periodogram appear also harmonics to the above mentioned periodicity of 6.7 , $5,4.1$ and 2.5 years.

3.5. Conclusions 
Both the wavelet analysis and the Lomb/Scargle periodogram, have identified the 27 days periodicity (with 13.5 days being its harmonic) in the dynamic parameters of the solar wind, Bx, By and the geomagnetic indices. The 1-1.4 years range of periodicities in the geomagnetic indices, the average IMF and solar wind speed and temperature was also identified. Furthermore the quasi-biennial (1.7-2.2 years) oscillation, along with its harmonics of 4 and 8 years, in all solar wind parameters, apart from IMF, and geomagnetic indices were detected. In solar cycle 22 the periodicities were more clearly defined than in the rest of the observation period, with well pronounced spectral peaks.

Acknowledgements This work was supported in part by the university of Athens research center (ELKE/EKPA). Christos Katsavrias thanks the LOC of the ESPM 13 for the financial support. The authors appreciate discussions with Alexander Hillaris. They also acknowledge many useful comments by the anonymous reviewer and the full attention of the editor.

\section{References}

Bai, T., Sturrock, P.A.: 1993, Evidence for a fundamental period of the sun and its relation to the 154 day complex of periodicities. Astrophys. J. 409, 476 - 486. doi $10.1086 / 172680$.

Bao, S.D., Zhang, H.Q.: 1998, Investigation of current helicities of active regions during the 22nd solar cycle. In: JOSO Annu. Rep., 1997, 132-133.

Benevolenskaya, E.E.: 1998, A Model of the Double Magnetic Cycle of the Sun. Astrophys. J. Lett. 509, L49-L52. doi $10.1086 / 311755$

Benevolenskaya, E.E.: 2000, A mechanism of helicity variations on the Sun. Solar Phys. 191, 247-255.

Cane, H.V., Richardson, I.G., von Rosenvinge, T.T.: 1998, Interplanetary magnetic field periodicity of 153 days. Geophys. Res. Lett. 25, 4437-4440. doi $10.1029 / 1998 G L 900208$.

Carta, F., Chlistovsky, F., Manara, A., Mazzoleni, F.: 1982, A comparative spectral analysis of the earth's rotation and the solar activity. Astron. Astrophys. $\mathbf{1 1 4}, 388-393$.

Clúa de Gonzalez, A.L., Gonzalez, W.D., Dutra, S.L.G., Tsurutani, B.T.: 1993, Periodic variation in the geomagnetic activity: A study based on the Ap index. J. Geophys. Res. 98, 9215-9232. doi:10.1029/92JA02200.

Currie, R.G.: 1976, Long period magnetic activity - 2 to 100 years. Astrophys. Spa. Sci. 39, 251-254. doi:10.1007/BF00640525.

Dimitropoulou, M., Moussas, X., Strintzi, D.: 2008, Enhanced Rieger-type periodicities' detection in X-ray solar flares and statistical validation of Rossby waves' existence. Mon. Not. Roy. Astron. Soc. 386, 2278-2284. doi $10.1111 / j .1365-2966.2008 .13203 . x$ 
Dimitropoulou, M., Moussas, X., Strintzi, D.: 2009, Enhanced Rieger type periodicities' detection in X-ray solar flares and statistical validation of Rossby waves' existence. In: N. Gopalswamy \& D. F. Webb (ed.) IAU Symposium, IAU Symposium 257, 159 - 163. doi $10.1017 /$ S1743921309029226.

Djurovic, D., Paquet, P.: 1996, The Common Oscillations of Solar Activity, the Geomagnetic Field, and the Earth's Rotation. Solar Phys. 167, 427-439. doi:10.1007/BF00146351.

Fenimore, E.E., Asbridge, J.R., Bame, S.J., Feldman, W.C., Gosling, J.T.: 1978, The power spectrum of the solar wind speed for periods greater than 10 days. J. Geophys. Res. 83, 4353-4357. doi:10.1029/JA083iA09p04353.

Gazis, P.R., Richardson, J.D., Paularena, K.I.: 1995, Long term periodicity in solar wind velocity during the last three solar cycles. Geophys. Res. Lett. 22, 1165 - 1168. doi $10.1029 / 95 G L 01017$.

Gonzalez, A.L.C., Gonzalez, W.D.: 1987, Periodicities in the interplanetary magnetic field polarity. J. Geophys. Res. 92, 4357-4375. doi $10.1029 /$ JA092iA05p04357.

Howe, R., Christensen-Dalsgaard, J., Hill, F., Komm, R.W., Larsen, R.M., Schou, J., Thompson, M.J., Toomre, J.: 2000, Deeply Penetrating Banded Zonal Flows in the Solar Convection Zone. Astrophys. J. Lett. 533, L163-L166. doi $10.1086 / 312623$

José Alves Bolzan, M., Roberto Rosa, R., Manuel Ramos, F., Roberto Fagundes, P., Sahai, Y.: 2005, Generalized thermostatistics and wavelet analysis of solar wind and proton density variability. Journal of Atmospheric and Solar-Terrestrial Physics 67, 1843-1851. doi 10.1016/j.jastp.2005.01.015

Katsavrias, C., Preka-Papadema, P., Moussas, X.: 2010, Study Of The Solar Wind Parameters During The 23rd Solar Cycle. In: A. Angelopoulos \& T. Fildisis (ed.) American Institute of Physics Conference Series, American Institute of Physics Conference Series 1203, 103-108. doi:10.1063/1.3322305.

Kiliç, H.: 2009, Short-Term Periodicities in Sunspot Activity and Flare Index Data during Solar Cycle 23. Solar Phys. 255, 155-162. doi:10.1007/s11207-008-9310-9

Krivova, N.A., Solanki, S.K.: 2002, The 1.3-year and 156-day periodicities in sunspot data: Wavelet analysis suggests a common origin. Astron. Astrophys. 394, 701-706. doi 10.1051/0004-6361:20021063.

Kudela, K., Mavromichalaki, H., Papaioannou, A., Gerontidou, M.: 2010, On Mid-Term Periodicities in Cosmic Rays. Solar Phys. 266, 173-180. doi:10.1007/s11207-010-9598-0

Laurenza, M., Vecchio, A., Carbone, V., Storini, M.: 2009, The quasi-biennial modulation of solar neutrino flux, solar and galactic cosmic rays by the solar cyclic activity. AGU Fall Meeting Abstracts, B9. 
Lean, J.L., Brueckner, G.E.: 1989, Intermediate-term solar periodicities - 100-500 days. Astrophys. J. 337, $568-578$. doi $10.1086 / 167124$.

Lomb, N.R.: 1976, Least-squares frequency analysis of unequally spaced data. Astrophys. Spa. Sci. 39, 447-462. doi:10.1007/BF00648343.

Lou, Y.-Q., Wang, Y.-M., Fan, Z., Wang, S., Wang, J.X.: 2003, Periodicities in solar coronal mass ejections. Mon. Not. Roy. Astron. Soc. 345, 809-818. doi: $10.1046 / j .1365-8711.2003 .06993 . x$

Mavromichalaki, H., Preka-Papadema, P., Petropoulos, B., Tsagouri, I., Georgakopoulos, S., Polygiannakis, J.: 2003, Low- and high-frequency spectral behavior of cosmic-ray intensity for the period 1953Â 1996. Annales Geophysicae 21, 1681-1689. doi 10.5194/angeo-21-1681-2003.

Morlet, J., Arens, G., Forgeau, I., Giard, D.: 1982, Wave Propagation and Sampling Theory. Geophysics 47, 203-236. doi 10.1190/1.1441328.

Mursula, K.: 1999, Simultaneous Occurrence of Mid-term Periodicities in Solar Wind Speed, Geomagnetic Activity and Cosmic Rays. In: D. Kieda, M. Salamon, and B. Dingus (ed.) IUPAP. Proceedings of the 26th International Cosmic Ray Conference 7, 123.

Mursula, K., Zieger, B.: 1996, The 13.5-day periodicity in the Sun, solar wind, and geomagnetic activity: The last three solar cycles. AGU 101, $27077-27090$.

Mursula, K., Usoskin, I., Zieger, B.: 1997, On the Claimed 5.5-year Periodicity in Solar Activity. Solar Physics 176, 201-210.

Obridko, V.N., Shelting, B.D.: 2007, Occurrence of the 1.3-year periodicity in the large-scale solar magnetic field for 8 solar cycles. Advances in Space Research 40, 1006 - 1014. doi $10.1016 /$ j.asr.2007.04.105.

Polygiannakis, J., Preka-Papadema, P., Moussas, X.: 2003, On signal-noise decomposition of time-series using the continuous wavelet transform: application to sunspot index. Mon. Not. Roy. Astron. Soc. 343, 725-734. doi $10.1046 / j .1365-8711.2003 .06705 . x$

Polygiannakis, J., Preka-Papadema, P., Petropoulos, B., Pothitakis, G., Moussas, X., Pappas, G., Hillaris, A.: 2002, Ephemeral periodicities in the solar activity. In: H. Sawaya-Lacoste (ed.) SOLMAG 2002. Proceedings of the Magnetic Coupling of the Solar Atmosphere Euroconference, ESA Special Publication 505, 537-540.

Prabhakaran Nayar, S.R., Radhika, V.N., Revathy, K., Ramadas, V.: 2002, Wavelet Analysis of solar, solar wind and geomagnetic parameters. Solar Phys. 208, $359-373$. 
Rieger, E., Kanbach, G., Reppin, C., Share, G.H., Forrest, D.J., Chupp, E.L.: 1984, A 154-day periodicity in the occurrence of hard solar flares? Nature 312, $623-625$. doi $10.1038 / 312623 a 0$.

Scargle, J.D.: 1982, Studies in astronomical time series analysis. II - Statistical aspects of spectral analysis of unevenly spaced data. Astrophys. J. 263, 835853. doi:10.1086/160554

Svalgaard, L., Wilcox, J.M.: 1975, Long-term evolution of solar sector structure. Solar Phys. 41, 461-475. doi 10.1007/BF00154083.

Torrence, C., Compo, G.P.: 1998, A Practical Guide to Wavelet Analysis. Bulletin of the American Meteorological Society 79, 61-78. doi:10.1175/1520-0477(1998)079;0061:APGTWA $i 2.0 . C O ; 2$.

Valdés-Galicia, J.F., Pérez-Enríquez, R., Otaola, J.A.: 1996, The Cosmic-Ray 1.68-Year Variation: a Clue to Understand the Nature of the Solar Cycle? Solar Phys. 167, 409-417. doi 10.1007/BF00146349.

Wolff, C.L.: 1992, 'Intermittent' solar periodicities. Solar Phys. 142, 187-195. doi:10.1007/BF00156641 
SOLA: WAVELETSolWinGeoINDICES_03c.tex; 31 October 2018; 5:58; p. 22 